Full length article

\title{
The effects of ethanol on the size-exclusion characteristics of type I dentin collagen to adhesive resin monomers
}

\author{
A. Chiba ${ }^{\text {a }}$, J. Zhou ${ }^{\text {b }}$, M. Nakajima ${ }^{\text {a }}$, J. Tan ${ }^{\text {b }}$, J. Tagami ${ }^{\text {a }}$, D.L.S. Scheffel ${ }^{\text {c }}$, J. Hebling ${ }^{\text {c }}$, K.A. Agee ${ }^{\text {, }}$, \\ L. Breschi ${ }^{\mathrm{e}}$, G. Grégoire ${ }^{\mathrm{f}}$, S.S. Jang ${ }^{\mathrm{g}}$, F.R. Tay ${ }^{\mathrm{d}}$, D.H. Pashley ${ }^{\mathrm{d}, *}$ \\ a Department of Cariology and Operative Dentistry, Tokyo Medical and Dental University, Tokyo, Japan \\ ${ }^{\mathrm{b}}$ Department of Prosthodontics, Peking University School and Hospital of Stomatology, Beijing, People's Republic of China \\ ${ }^{c}$ Department of Orthodontics and Pediatric Dentistry, Universidade Estadual Paulista-UNESP, Araraquara Dental School, Araraquara, Sao Paulo, Brazil \\ ${ }^{\mathrm{d}}$ Department of Oral Biology, The Dental College of Georgia, Augusta University, Augusta, GA, USA \\ e Department of Biomedical and Neuromotor Sciences DIBINEM, University of Bologna and IGM-CNR Unit of Bologna, Bologna, Italy \\ ${ }^{\mathrm{f}}$ Department of Biomaterials, Faculty of Odontology, University of Toulouse III, Toulouse, France \\ ${ }^{\mathrm{g}}$ Department of Materials Science Engineering, Georgia Institute of Technology, Atlanta, GA, USA
}

\section{A R T I C L E I N F O}

\section{Article history:}

Received 8 October 2015

Received in revised form 4 January 2016

Accepted 27 January 2016

Available online 28 January 2016

\section{Keywords:}

Bound water

Collagen

Dentin

Ethanol-wet bonding

Polar solvents

Mineralized collagen

Size-exclusion

Resin-dentin bonding

\begin{abstract}
A B S T R A C $T$
During dentin bonding with etch-and-rinse adhesive systems, phosphoric acid etching of mineralized dentin solubilizes the mineral crystallites and replaces them with bound and unbound water. During the infiltration phase of dentin bonding, solvated adhesive resin comonomers are supposed to replace all of the unbound collagen water and polymerize into copolymers. A recently published review suggested that dental monomers are too large to enter and displace water from tightly-packed collagen molecules. Conversely, recent work from the authors' laboratory demonstrated that HEMA and TEGDMA freely equilibrate with water-saturated dentin matrices. However, because adhesive blends are solvated in organic solvents, those solvents may remove enough free water to allow collagen molecules to come close enough to exclude adhesive monomer permeation. The present study analyzed the size-exclusion characteristics of dentin collagen, using a gel permeation-like column chromatography technique, filled with dentin powder instead of Sephadex beads as the stationary phase. The elution volumes of different sized test molecules, including adhesive resin monomers, studied in both watersaturated dentin, and again in ethanol-dehydrated dentin powder, showed that adhesive resin monomers can freely diffuse into both hydrated and dehydrated collagen molecules. Under these in vitro conditions, all free and some of the loosely-bound water seems to have been removed by ethanol. These results validate the concept that adhesive resin monomers can permeate tightly-bound water in ethanol-saturated collagen molecules during infiltration by etch-and-rinse adhesives.
\end{abstract}

\section{Statement of Significance}

It has been reported that collagen molecules in dentin matrices are packed too close together to allow permeation of adhesive monomers between them. Resin infiltration, in this view, would be limited to extrafibrillar spaces. Our work suggests that monomers equilibrate with collagen water in both water and ethanol-saturated dentin matrices.

(c) 2016 Acta Materialia Inc. Published by Elsevier Ltd. All rights reserved.

\section{Introduction}

The distribution of bound and unbound (free) water in completely demineralized dentin matrices was found to be about

\footnotetext{
* Corresponding author at: Department of Oral Biology, The Dental College of Georgia, Augusta University, Augusta, GA 30912-1129, USA

E-mail address: dpashley@gru.edu (D.H. Pashley).
}

$25 \mathrm{wt} \%$ and $75 \mathrm{wt} \%$, respectively [1]. Water that is tightly bound to dentin matrices should be regarded as structural water. It is bound so tightly that it does not behave like regular free water [2]. That is, tightly-bound water cannot be evaporated at atmospheric pressure and body temperature. It cannot diffuse into water-free but water-miscible solvents like ethanol [1]. Presumably, when adhesive monomers infiltrate into demineralized dentin, they diffuse over and around tightly-bound water [1]. 
Fortunately, three-quarters of the total water in dentin is unbound and can be removed by evaporation and/or chemical dehydration using water-miscible, water-free organic solvents [1].

If only half of the unbound water is removed during the 60-90 s used by most dentists to bond resins to dentin, then up to half of the collagen matrix will remain infiltrated by water rather than resin. That water is used by the endogenous proteases of dentin to slowly hydrolyze collagen fibrils, solubilizing insoluble collagen and causing decreases in resin-dentin bond strength over time $[3,4]$. The more free water that can be removed from demineralized dentin, the more adhesive resin monomers can be taken up by the matrix during resin infiltration. The key to creation of ideal resin-dentin bonds is to replace all unbound water with water-free, but watermiscible solvents like ethanol [5] that can solvate adhesive resin monomers. The most tightly bound is the first layer that directly hydrogen bonds to collagen [1]. The second layer is firmly bound and is covered by the least tightly bound water on the outside of collagen. When adhesive monomers infiltrate into the matrix, they can only interact with the outermost, loosely bound water.

In a study on the limitations to resin bonding at the nanometer scale [6], the authors argued that the packing density of hydrated collagen molecules in the collagen fibrils of demineralized dentin is so dense, there is insufficient space for the uptake of adhesive monomers like 2-hydroxyethyl methacrylate (HEMA) or triethylene glycol dimethacrylate (TEGDMA). They implied that collagen molecules were covered with bound, structural water that is necessary for the stability of collagen, and that there was too little space available between collagen molecules for resin uptake. A recent ${ }^{1} \mathrm{H}$ NMR report indicated that resin infiltration of ethanolrinsed demineralized dentin by EXCITE ${ }^{\circledR}$ (Ivoclar-Vivadent, Schaan, Lichtenstein, an adhesive containing $50 \mathrm{vol} \%$ ethanol) removed free water but not all bound water [7]. This suggests that solvated adhesive resin monomers can diffuse down to the nanoscopic level in collagen fibrils where bound water resides, and that it may remove some loosely-bound water, but leaves most of the tightly-bound water in place. Those authors [7] speculated that bound water holds intermolecular spaces between collagen molecules open for adhesive resin monomer uptake. This suggests that adhesive resin monomers, especially water-insoluble dimethacrylates, may partially permeate between collagen molecules by diffusing over collagen's most peripheral, loosely bound water that was partially replaced by ethanol.

To measure the size inclusion or exclusion characteristics of insoluble type I collagen, Toroian et al. [8] filled $25 \times 1.25 \mathrm{~cm}$ diameter columns with mineralized bone powder to conduct gel permeation-like experiments. In gel-permeation column chromatography, a column is filled with hydrated polymeric beads, where more than half the total water of the column resides inside the beads, while the rest resides outside the beads. When large molecules such as albumin are applied, they are too large to enter the polymer beads, hence they elute from the column quickly in the first few collected fractions (Fig. 1). Small molecules such as glucose or phosphate easily penetrate the hydrated beads. Their elution is delayed because they equilibrate with intrabead freewater. Since there was little free water in mineralized bone, none of the small (or large) tracers were delayed in their transit through the column. They all eluted from the column rapidly in the void volume. However, when they completely demineralized the bone powder, large amounts of water (60-70 vol\%) replaced the original mineral volume. Some of that water was bound, but much of it was unbound. When they applied tracer molecules of increasing size to columns filled with demineralized bone powder, they found that large molecules, such as albumin (67 kDa), were too large to permeate into "collagen water" and were eluted quickly. Intermediate-size molecules like calcitonin $(5.7 \mathrm{kDa})$ were delayed somewhat in their elution, while small molecules like glucose
(180 Da) and phosphate were even more delayed in their elution, indicating that they equilibrated with what is presumed to be unbound collagen water.

That study was repeated on dentin collagen by Takahashi et al. [9]. Their results were similar to those of Toroian et al. [8], but they used dentin powder instead of bone powder, and included the dental adhesive resin monomers 2-hydroxyethyl methacrylate (HEMA) and triethyleneglycol dimethacrylate (TEGDMA) in their small molecular tracers. The authors showed that watersaturated, completely demineralized dentin powder equilibrated with HEMA and TEGDMA, and that both monomers were delayed in their elution, indicating that those monomers equilibrated with collagen water. This suggests that there may be sufficient space between collagen molecules for resin uptake. However, adhesive resin monomers are solvated in ethanol, not water. When applied to dentin, these solvents may remove intracollagen free water by chemical dehydration and allow the collagen molecules to come closer together, excluding monomer uptake. Thus, the experiments of Takahashi et al. [9] need to be repeated on columns of demineralized dentin powder that are equilibrated with ethanol, to determine if dimethacrylates such as TEGDMA or bisphenol A glycidyl dimethacrylate (BisGMA) can enter the ethanol-solvated volume of dehydrated dentin matrix collagen, or if they are excluded. Accordingly, the purpose of the present work was to determine if TEGDMA and BisGMA, as examples of small, relatively hydrophobic adhesive dimethacrylate monomers, can equilibrate with ethanol within collagen molecules in demineralized dentin powder. The null hypothesis tested was that TEGDMA and BisGMA cannot equilibrate with ethanol-solvated dentin collagen.

\section{Materials and Methods}

\subsection{Materials}

Blue Dextran $(2 \times 106 \mathrm{Da})$ was obtained from Sigma/Aldrich (St. Louis, MO, USA), and was used as an example of a relatively large water-soluble tracer. HEMA (130 Da), TEGDMA (286 Da) and BisGMA (512 Da) were obtained from ESSTECH (Essington, PA, USA).

\subsection{Creation of dentin powder}

Extracted bovine incisors were obtained from a local abattoir. Six hundred freshly extracted bovine incisor teeth, which were stored in $0.9 \% \mathrm{NaCl}$ containing $0.02 \%$ sodium azide at $4{ }^{\circ} \mathrm{C}$ to prevent bacterial growth, were used in the present study. Dentin devoid of enamel, cementum and pulpal tissues were prepared from those teeth using dental burs with a high speed hand piece with copious air-water spray. Using an Isomet saw (Buehler Ltd., Lake Bluff, IL, USA) under water cooling, the dentin specimens were cut into four equal-sized fragments. The resulting tooth fragments were dehydrated in acetone for $20 \mathrm{~min}$ and placed in liquid nitrogen for $15 \mathrm{~min}$. The frozen dentin fragments were then reduced to dentin powder in a Wiley Mini Mill (Thomas Scientific, Model 3383-L10, Swedesboro, NJ, USA). The resulting powder was passed through a series of stacked sieves (Nos. 18, 30, 50, 140; Cole-Palmer, Vernon Hills, IL, USA). The powder that passed through $300 \mu \mathrm{m}$ sieves but was retained on $106 \mu \mathrm{m}$ sieves, was used to fill the column. The mineralized dentin powder was kept frozen at $-80^{\circ} \mathrm{C}$ until use, to prevent degradation of the collagen component.

\subsection{Gel filtration procedures}

A $1 \times 30 \mathrm{~cm}$ glass column was filled with $25 \mathrm{~cm}^{3}$ of mineralized dentin powder and equilibrated with $20 \mathrm{mM}$ Tris buffer ( $\mathrm{pH} 7.4$ ) 


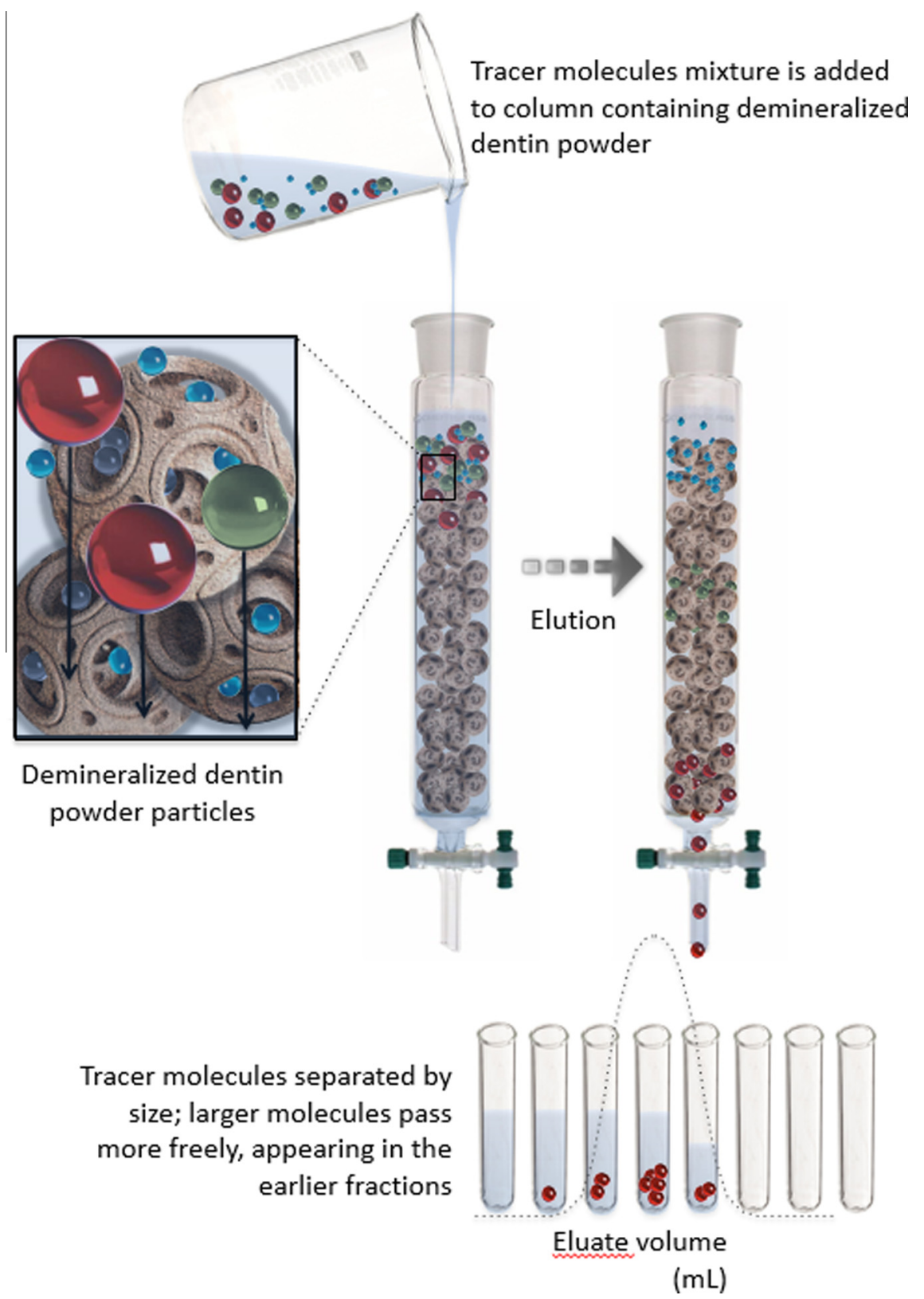

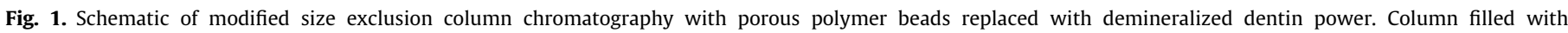

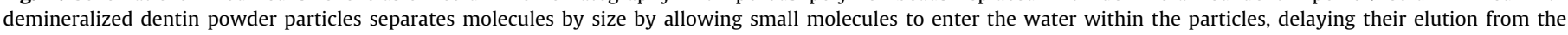
column (modified after Lehninger, Principles of Biochemistry by David L. Nelson and Michael M. Cox, 5th ed., W.H. Freeman and Company, New York, with permission).

that contained $0.15 \mathrm{M} \mathrm{NaCl}$. That buffer was used to elute the column at a flow rate of $14.4 \mathrm{~mL} / \mathrm{h}$ until the final effluent absorbance at $220 \mathrm{~nm}$ was less than 0.1 , indicating that all extrafibrillar protein had been rinsed from the column. Sample volumes were 1.0 $( \pm 0.1) \mathrm{mL}$ and were collected in a fraction collector set to change every 5 min.

Blue dextran $(4 \mathrm{mg} / \mathrm{mL})$ and HEMA $(0.125 \mathrm{mg} / \mathrm{mL})$ were diluted in buffer and $500 \mu \mathrm{L}$ of each tracer were separately put on the top of the column. A syringe pump (Harvard Apparatus, Holliston, MA, USA) provided a constant $14.4 \mathrm{~mL} / \mathrm{h}$ flow of buffer. The eluate from the column passed into a fraction collector and was collected in individual tared tubes. The volume of each fraction $(1.0 \pm 0.1 \mathrm{~mL})$ was determined by weighing the tubes and dividing by the density of the buffer $(1.001 \mathrm{~g} / \mathrm{mL})$. The absorbance of each fraction was read by a spectrophotometer (Shimadzu Scientific Instruments,
Inc., Columbia, MD, USA) at 620 (Blue Dextran) or 220 (HEMA) $\mathrm{nm}$. Each tracer was repeated three times and the average absorbance was plotted against the total elution volume.

After running the tracers through the buffer-saturated mineralized powder, the column was unpacked and the powder completely demineralized in 10\% phosphoric acid ( $\mathrm{pH} 1.0)$ for $24 \mathrm{~h}$ at $25{ }^{\circ} \mathrm{C}$ under stirring. Complete demineralization was tested as follows: After $24 \mathrm{~h}$ of demineralization, the dentin powder was resuspended in fresh demineralizing solution $(\mathrm{pH} 1.0)$ for $1 \mathrm{~h}$. After allowing the powder to settle, $1 \mathrm{~mL}$ of the supernatant was mixed with $1 \mathrm{~mL}$ of $2.7 \mathrm{wt} \%$ monopotassium monohydrogen oxalate. The absence of white precipitate indicated there was no ionized calcium in the medium. Positive controls were the addition of $1 \mathrm{~mL}$ of $0.2 \mathrm{M} \mathrm{CaCl}_{2}$ with $1 \mathrm{~mL}$ of the same half-neutralized oxalic acid to produce a heavy white precipitate of calcium oxalate. 
The completely demineralized dentin powder was then repacked into the column. Extra demineralized powder was added to compensate for the volume lost during the demineralization process, in order to keep the original height of the column. Tris buffer ( $\mathrm{pH}$ 7.4) was pumped through the packed powder for three days, until elution absorbance fell to below 0.1 at $220 \mathrm{~nm}$.

Tracer samples were dissolved in aqueous buffer (Table 1 ) and passed through the buffer-saturated demineralized powder as described for mineralized dentin. Then, the column was once more unpacked and the excess buffer removed from the powder using a sieve (Cole Parmer No. 140) and absorbent paper. The powder was saturated with $100 \%$ ethanol under stirring for $1 \mathrm{~h}$. The excess ethanol was removed, and the column was re-packed with fresh $100 \%$ ethanol and rinsed at flow rate $14.4 \mathrm{~mL} / \mathrm{h}$ for $24 \mathrm{~h}$ to remove any residual free water. Tracers were dissolved in ethanol (Table 1) and passed through the ethanol-saturated demineralized column as previously described.

Because the adhesive resin monomer BisGMA is not soluble in buffer but is soluble in ethanol, it was added to the ethanol tracers. Blue Dextran, insoluble in $100 \%$ ethanol, was first dissolved in a small amount of water and then diluted by ethanol to obtain a final concentration of $0.5 \mathrm{mg} / \mathrm{mL}$ in $4 \%$ water/96\% ethanol. Due to the high evaporative nature of ethanol, each eluted fraction was capped immediately after collection. The elution of water or ethanol-soluble test tracers was spectrophotometrically analyzed as follows: Blue Dextran, absorbance at $620 \mathrm{~nm}$ [9]; TEGDMA, BisGMA and HEMA, absorbance at $220 \mathrm{~nm}$ [9].

\subsection{Statistical analysis}

Because of the imbalance concerning tracers, dentin condition (mineralized vs. demineralized) and mobile phase (buffer vs. ethanol), it was not possible to subject the data to a two-way analysis of variance. Therefore, data from elution volumes were submitted to multiple one-way ANOVA, complemented by Tukey's test for pairwise comparisons, or multiple t-tests using SPSS 23.0 (SPSS Inc., Chicago, IL, USA), at a $95 \%$ level of confidence.

One column was used multiple times. That is, the elution volumes of the various tracers were measured in mineralized dentin powder, then they were measured again in demineralized dentin equilibrated with aqueous buffer, and then again in demineralized dentin powder equilibrated with ethanol. Each tracer was measured three times in the same column except for BisGMA which is not soluble in aqueous buffer. When a column received an irreversible treatment such as being treated with a crosslinking agent, the tracers are used again three times ( $n=3$, Table 2$)$ and then the column was discarded.

Table 1

Tracer concentration $(\mathrm{mg} / \mathrm{ml})$ for each mobile phase and their respective wavelength $(\mathrm{nm})$.

\begin{tabular}{llll}
\hline Tracer & MW (Da) & $\begin{array}{l}\text { Dentin powder } \\
(106 \mu \mathrm{m}<\text { particle } \\
\text { size }<300 \mu \mathrm{m})\end{array}$ & \\
\cline { 3 - 4 } & & Tracer concentration $(\mathrm{mg} / \mathrm{mL})$ \\
\cline { 3 - 4 } & & Buffer & Ethanol \\
\hline Blue Dextran ${ }^{* *}$ & $2,000,000$ & 4.0 & 0.5 \\
BisGMA & 512 & Not soluble & 0.15 \\
TEGDMA & 286 & 0.07 & 0.25 \\
HEMA & 130 & 0.125 & 0.5 \\
\hline
\end{tabular}

Abbreviations: BisGMA: bisphenol A glycidyl dimethacrylate; TEGDMA: triethylene glycol dimethacrylate; HEMA: 2-hydroxyethyl methacrylate.

Since Blue Dextran is not soluble in ethanol, this tracer was solubilized in water and then diluted with ethanol to a final concentration of $4 \%$ water $/ 96 \%$ ethanol.

** Blue Dextran absorbance was read at $620 \mathrm{~nm}$ while all the other tracers were read at $220 \mathrm{~nm}$.

\section{Results}

3.1. Size-exclusion characteristics of mineralized bovine dentin powder using aqueous buffer as the mobile phase

The elution of large (Blue Dextran) and small (HEMA) tracers from columns of mineralized dentin powder produced elution volumes that were very similar to those reported by Takahashi et al. [9]. That is, both blue dextran and HEMA eluted at $11.9 \pm 0.5 \mathrm{~mL}$ and $12.5 \pm 0.2 \mathrm{~mL}$, with no significant difference between the two groups ( $t$-test; $p=0.113$ ). These values were similar to the void volume of the column (Table 2, Fig. 2).

\subsection{Size-exclusion characteristics of completely demineralized bovine dentin powder using aqueous buffer as the mobile phase}

Application of the large molecule Blue Dextran resulted in an elution volume of $11.4 \pm 0.2 \mathrm{~mL}$, which was not significantly different from the mineralized powder Blue Dextran elution value ( $p>0.05$; Tukey's test, Row 1 in Table 2). The elution of HEMA at $18.4 \pm 0.4 \mathrm{~mL}$ (Table 2, Fig. 3) represented a shift to the right when compared to the mineralized elution volume. Similar to HEMA, the small adhesion resin monomer TEGDMA had an elution volume of $18.5 \pm 0.02 \mathrm{~mL}$. Elution volumes for Blue Dextran, HEMA and TEGDMA with the use of buffer as the mobile phase indicated significant difference among the three groups $(p<0.001$; Column 2 in Table 2). The elution volumes of HEMA and TEGDMA were not significantly different from one another (Tukey's test, $p>0.05$ ), but were both significantly higher than that of Blue Dextran (Tukey's test, $p<0.05$ for both comparisons).

\subsection{Size exclusion characteristics of completely demineralized dentin powder using $96 \%$ ethanol as the mobile phase}

Because Blue Dextran is not soluble in $100 \%$ ethanol, the mobile phase was replaced with $4 \%$ water $/ 96 \%$ ethanol. The elution volumes of Blue Dextran and HEMA in ethanol-saturated dentin powder were higher than were seen in water-saturated demineralized dentin powder (Fig. 3). Specifically, the elution volume of Blue Dextran was $11.4 \pm 0.2 \mathrm{~mL}$ and HEMA was $18.4 \pm 0.4 \mathrm{~mL}$ in water-saturated dentin, but were $12.8 \pm 0.4 \mathrm{~mL}$ and $19.3 \pm 0.2 \mathrm{~mL}$, respectively, in ethanol-saturated dentin (Table 2, Fig. 3). The elution volumes of TEGDMA was $18.5 \pm 0.02 \mathrm{~mL}$ and $15.9 \pm 0.3 \mathrm{~mL}$ in water-saturated vs ethanol-saturated demineralized dentin, respectively. All of these shifts were significantly different $(p<0.05)$. One-way ANOVA of the elution volumes for Blue Dextran, BisGMA, HEMA and TEGDMA with the use of ethanol as the

Table 2

Elution volume $(\mathrm{mL})$ of different tracers diffused through mineralized and demineralized bovine dentin powder.

\begin{tabular}{llllr}
\hline Tracer & MW (Da) & \multicolumn{2}{l}{$\begin{array}{l}\text { Dentin powder }(106 \mu \mathrm{m}<\text { particle } \\
\text { size }<300 \mu \mathrm{m})\end{array}$} \\
\cline { 3 - 5 } & & Mineralized & Demineralized \\
\cline { 3 - 5 } & & Buffer & \multicolumn{1}{c}{ Buffer } & Ethanol \\
\hline Blue Dextran & $2,000,000$ & $11.9 \pm 0.5^{\text {AB.a }}$ & $11.4 \pm 0.2^{\text {B.b }}$ & $12.8 \pm 0.4^{\text {A.d }}$ \\
BisGMA & 512 & Not soluble & Not soluble & $18.1 \pm 0.2^{\text {b }}$ \\
TEGDMA & 286 & - & $18.5 \pm 0.02^{\text {A.a }}$ & $15.9 \pm 0.3^{\text {B.c }}$ \\
HEMA & 130 & $12.5 \pm 0.2^{\text {C.a }}$ & $18.4 \pm 0.4^{\text {B.a }}$ & $19.3 \pm 0.2^{\text {A.a }}$ \\
\hline
\end{tabular}

Numbers are mean \pm standard deviation $(n=3)$ in milliliters $(\mathrm{mL})$.

Abbreviations: BisGMA: bisphenol A glycidyl dimethacrylate; TEGDMA: triethylene glycol dimethacrylate; HEMA: 2-hydroxyethyl methacrylate.

Upper case letters represent comparisons in the horizontal lines while lower case letters represent comparisons in the vertical columns. Means identified with the same letter are not statistically different (Tukey, $p>0.05$ ). 


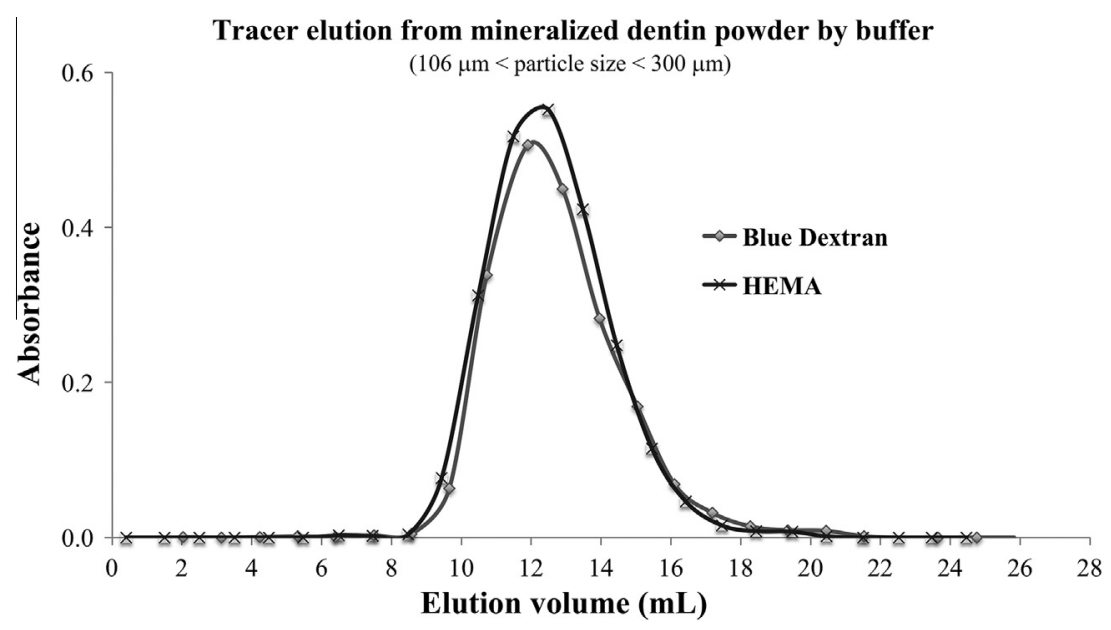

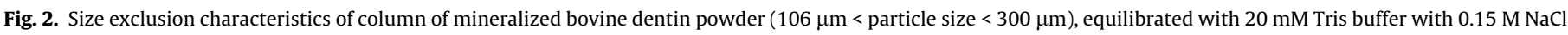

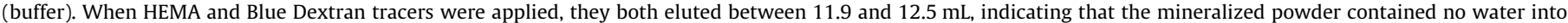
which small tracers like HEMA could diffuse.

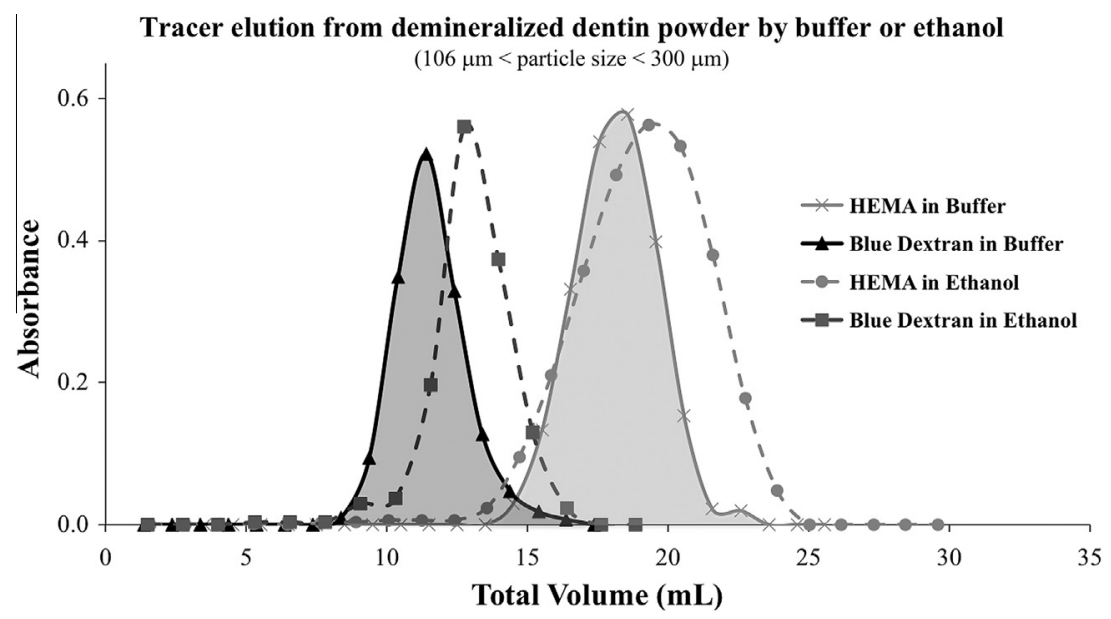

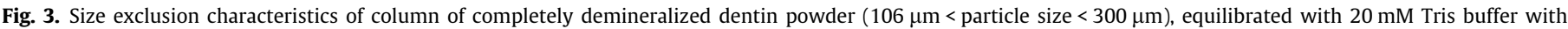

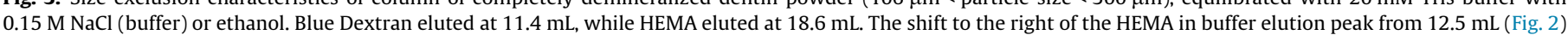

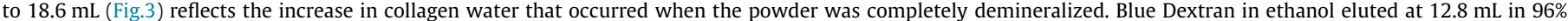

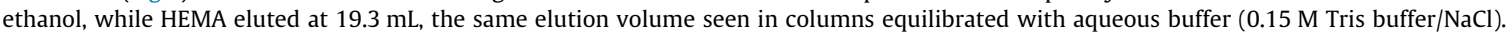

mobile phase indicated significant difference among the four groups ( $p<0.001$; Column 3 in Table 2). Those elution volumes were all significantly different from one another, in the order: HEMA $\quad(19.3 \pm 0.2 \mathrm{~mL})>$ BisGMA $\quad(18.1 \pm 0.2 \mathrm{~mL})>$ TEGDMA $(15.9 \pm 0.3 \mathrm{~mL})>$ Blue Dextran $(12.8 \pm 0.4 \mathrm{~mL})$ (Tukey's test, $p<0.05$ for all comparisons).

\section{Discussion}

The initial control experiments were done to determine if there was a measurable volume of water in hydrated mineralized dentin. Results from Section 3.1 may be interpreted by the fact that collagen in the mineralized dentin matrix is covered by apatite crystallites, and that there is little free water (with the exception of water in dentinal tubules) available in the stationary phase. Blue Dextran, with a molecular weight of $2 \times 10^{6} \mathrm{Da}$, was too large to enter any of the mineralized collagen fibrils in the mineralized dentin powder. It could enter the interstitial spaces between the dentin powder particles. However, HEMA, with a molecular weight of only $130 \mathrm{Da}$, should be able to diffuse into collagen water, but only if it was demineralized. It is thought that in the mineralized state, all collagen water has been displaced by apatite mineral crystallites $[10,11]$. During mineralization, water provides the aqueous environment for infiltration of amorphous calcium phosphate precursors into collagen fibrils to initiate nucleation and growth of intrafibrillar apatite crystallites within the collagen fibril. As mineralization proceeds, the loosely-bound and bulk free water of the collagen matrix is progressively replaced by the intrafibrillar crystallites within the collagen fibrils and extrafibrillar apatite crystallites along the interfibrillar spaces [12]. The fact that the mean elution volume of HEMA (130 Da) was $12.5 \pm 0.2 \mathrm{~mL}$ while that of Blue Dextran $\left(2 \times 10^{6} \mathrm{Da}\right)$ is $11.9 \pm 0.5 \mathrm{ml}$ suggests that there was little water in the mineralized collagen matrix $[8,9]$.

In marketed dental adhesives, both TEGDMA and BisGMA are often solubilized in HEMA because they are so much more soluble in HEMA than water. We did not do that in the current experiments because the absorbance of HEMA would have interfered with the absorbance of TEGDMA at the same wavelength. When the size exclusion characteristics of these adhesive resin monomer molecules were examined using demineralized dentin powder as the stationary phase and aqueous buffer as the mobile phase, there 
was a shift in the elution volume of HEMA to the right when compared to the mineralized elution volume (see Figs. 2 and 3). The numerical results presented in Section 3.2 (Table 2) indicate that smaller tracers such as HEMA and TEGDMA had diffused into collagen water and had been delayed in its elution, when compared to Blue Dextran, the largest tracer molecule tested.

When the aqueous buffer was replaced with $100 \%$ ethanol to remove all free water and some of the most loosely-bound water, TEGDMA and BisGMA equilibrated with the elution volumes of ethanol-solvated demineralized dentin, requiring rejection of the test null hypothesis that "TEGDMA and BisGMA cannot equilibrate with ethanol-solvated dentin collagen". The elution volumes of HEMA $(19.3 \pm 0.2 \mathrm{~mL})$, TEGDMA $(15.9 \pm 0.3 \mathrm{~mL})$ and BisGMA $(18.1 \pm 0.2 \mathrm{~mL}$ ) were all between 15.9 and $19.3 \mathrm{~mL}$ (Section 3.2, Table 2), indicating that they could enter ethanol-solvated demineralized dentin matrices to variable extents.

The findings that TEGDMA and BisGMA equilibrated with elution volumes that were significantly larger than those of Blue Dextran have important clinical implication, in that these monomers are not excluded from ethanol-dehydrated dentin. We speculate that ethanol can remove all free water from demineralized dentin powder and some loosely-bound water and permeate around collagen peptides in completely demineralized dentin which are held apart by firmly bound water. This allows ethanol-solvated dimethacrylates to diffuse over and around collagen-bound water without disturbing it.

In the present study, $4 \%$ water was in the ethanol eluate to allow Blue Dextran, the only large tracer that was soluble in both water and ethanol, to stay in solution. Ethanol can hydrogen bond to loosely-bound water. This may have increased the elution volumes of the small tracers enough to be significantly different. An important observation is that replacing most of the water in the mobile phase with ethanol did not shift the elution volumes to the left of their aqueous volumes. Future experiments will have to be performed using acetone, a polar solvent that cannot hydrogen bond to demineralized collagen matrices.

We speculate that bound water protects and stabilizes the collagen triple helix but allows ethanol-solvated monomers to come into close proximity with the collagen molecules. The recent saturation transfer difference (STD) NMR studies by Hiraishi et al. [13] concluded that molecular-level interactions of HEMA did not occur with atelocollagen solvated in dimethyl sulphoxide. The authors concluded that the layer of water on collagen prevents molecular level interactions of HEMA with collagen. These results were opposite to the earlier computer molecular modeling reports by Vaidyanathan and Vaidyanathan [14] on HEMA docking within collagen triple helices, which was done on molecules free of bound water.

In the present study, a large excess of ethanol was used to remove free and most of the loosely-bound water. Thus, the degree of removal of these two categories of water from the demineralized matrix was probably far more thorough than is possible during the relatively short treatment times used in clinical resin-dentin bonding. The fact that we never observed size-exclusion of any common dental dimethacrylate monomers indicates that under these extreme dehydrated conditions, there is sufficient tightly-bound water and residual solvent to permit equilibration of dimethacrylates with demineralized dentin. Grégorie et al. [7] published ${ }^{1} \mathrm{H}$ NMR scans of ethanol-rinsed resin-bonded dentin that indicated tightly-bound water remains in resin-dentin bonds even after rinsing with ethanol. Any excess residual free water within or around collagen would prevent permeation of TEGDMA, BisGMA or UDMA as they are almost insoluble in water. Pretreatment of watersaturated dentin by water-free but water-miscible ethanol to remove free water would seem to be a prudent approach for improving comonomer infiltration during resin-dentin bonding. Contemporary bonding practices make too many compromises in an attempt to "save time" [15]. The cost of saving time may be too much residual water remains in resin-dentin bonds, which results in poor durability of these bonds over time by allowing water to fuel hydrolysis of collagen by endogenous dentin proteases [3].

There are alternative explanations for why the tracer elution volumes of most of the adhesive monomers shifted to the right instead of to the left when the demineralized dentin powder was dehydrated with ethanol. When pure ethanol was pumped through a water-saturated column of demineralized dentin, voids began to appear in the column. These voids may have represented nitrogengas bubbles coming out of solution when ethanol encountered nitrogen-saturated water.

To eliminate $\mathrm{N}_{2}$ gas-induced voids in the column, we unloaded the column and slowly replaced water with ethanol in a beaker with constant stirring until all nitrogen had been allowed to escape, and then repacked the column with ethanol-saturated dentin powder.

To avoid having buffer salts come out of solution when the column was equilibrated with ethanol, we eluted the column with pure water for $8 \mathrm{~h}$ to remove all buffer salts before switching to ethanol. We then unpacked the column into a beaker and replaced the water with multiple batches of ethanol under constant stirring to allow gradual exchange of water by ethanol. Then the column was repacked and the ethanol experiment continued. Thus, under these conditions, we did not see any generation of voids or agglomerations when ethanol was used as the eluate.

Another possibility would be aggregation or hydrogen bonding of collagen particles together to form agglomerations. This cannot occur in aqueous buffer, because water preferentially hydrogen bonds with carbonyl and amide nitrogen moietites. Such as a phenomenon theoretically can occur in the absence of water, when alcohol replaces free water in the column. Arguing against that idea is when we purposely covalently cross-linked collagen with $0.5 \mathrm{M}$ carbodiimide, there was no change in the elution volume of our tracers (data not shown). Thus, we prescreened all of our tracer molecules to avoid any adverse interactions with collagen under normal operating conditions.

The observation that ethanol dehydration of demineralized dentin powder caused the elution volumes of BisGMA and HEMA to be $18-19 \mathrm{~mL}$, while that of TEGDMA fell from $18.5 \mathrm{~mL}$ to $15.9 \mathrm{~mL}$ seems paradoxical. When the water-saturated column was dehydrated by equilibrating the dentin matrix powder with ethanol, the elution volumes of BisGMA and HEMA increased while that of TEGDMA decreased (Table 2). The following speculation is offered as an explanation.

Molecules interact with each other based on hydrophobic or dispersive forces, as well as polar forces and hydrogen bonding cohesive forces [16]. The relative contribution of these three components to the total cohesive energy of molecules can be described by the triple solubility parameters of Hansen [16] or Hoy [17]. Water has such a high chemical concentration $(55 \mathrm{~mol} / \mathrm{L})$ and is such a strong hydrogen bonding solvent, that its solubility parameter for hydrogen bonding $\left(40 \mathrm{~J} / \mathrm{cm}^{3}\right)^{1 / 2}$ tends to overpower the dispersive and polar components (Table 3 ). When dentin matrices are saturated with water, water molecules hydrogen bond to carbonyl oxygen and amide nitrogen moieties in collagen peptides. This prevents interpeptide hydrogen bonding (H-bonding) between adjacent peptides. Monomers like TEGDMA and HEMA can produce weak H-bonding to loosely-bound water in collagen [18]. However, when water is replaced by ethanol, there is less interpeptide $\mathrm{H}$-bonding of water to collagen, although ethanol can $\mathrm{H}$-bond to collagen albeit less strongly $\left(\delta_{\mathrm{h}}\right.$ ethanol $=20(\mathrm{~J} /$ $\mathrm{cm}^{3} / /^{1 / 2}$ ).

Under these conditions, the solubility parameters for polar forces may become a significant influence on monomer interaction 
Table 3

Hoy's solubility parameters of adhesive monomers.

\begin{tabular}{lllrl}
\hline Monomer & $\delta_{\mathrm{d}}$ & $\delta_{\mathrm{p}}$ & \multicolumn{1}{c}{$\delta_{\mathrm{h}}$} & $\delta_{\mathrm{t}}$ \\
\hline BisGMA & 16.6 & 13.4 & 5.8 & 22.1 \\
TEGDMA & 14.2 & 10.1 & 8.2 & 19.2 \\
HEMA & 13.3 & 12.3 & 15.2 & 23.6 \\
\hline
\end{tabular}

Values are all $\left(\mathrm{J} / \mathrm{cm}^{3}\right)^{1 / 2}$. Abbreviations: BisGMA: 1:2 addition product of bisphenylA diglycidyl ether and methacrylic acid; TEGDMA: triethylene glycol dimethacrylate; HEMA: 2-hydroxyethyl methacrylate; $\delta_{\mathrm{d}}$ : Hoy's solubility parameter for dispersive forces; $\delta_{\mathrm{p}}$ : Hoy's solubility parameter for polar forces; $\delta_{\mathrm{h}}$ : Hoy's solubility parameter for hydrogen bonding forces; $\delta_{\mathrm{t}}$ : Hoy's solubility parameter for total cohesive forces.

in dehydrated collagen (dry collagen, Table 2, [18]) because BisGMA and HEMA have larger polar and total solubility parameters than does TEGDMA (Table 3). Thus, BisGMA and HEMA would interact more with collagen than would TEGDMA, causing them to be more delayed in their elution from the size-exclusion column, than would TEGDMA. That is, TEGDMA would be eluted before BisGMA and HEMA, but only under dehydrated (i.e. ethanolsaturated) conditions. Future molecular dynamic simulations may be useful in testing this speculation.

We speculate that if adhesive monomers dissolved in ethanol can infiltrate over ethanol-saturated collagen peptides and their associated noncollagenous molecules, including endogenous proteases, it may be possible to incorporate hydrophobic adhesive resins $[19,20]$ into the active sites of those proteases to inactivate their activity at the same time resins are infiltrating around collagen molecules. Such resin-dentin bonds should be far more durable than are contemporary resin-dentin bonds $[21,22]$. Clearly, more research is needed to characterize resin-dentin infiltration at the nanoscale.

\section{Disclosures}

None of the authors of this study received any corporate support for the use of products used in this work. None of the authors have any conflicts of interest with the products used in the study.

\section{Acknowledgments}

This work was supported, in part, by R01 DE015306 from the NIDCR to DP (PI). The authors are grateful to Mrs. Michelle Barnes for her secretarial support, and to ESSTECH Inc. (Essington, PA) for adhesive monomers, and to PFL Foods Augusta, GA for donating bovine incisors. The authors also thank Sao Paulo Research Foundation - FAPESP for financial support (Process 2014/17232-4 and 2014/18160-7).

\section{References}

[1] K.A. Agee, A. Prakki, T. Abu-Haimed, G.H. Naguib, M.A. Nawareg, A. TezvergilMutluay, D.L.S. Scheffel, C. Chen, S.S. Jang, H. Hwang, M. Brackett, G. Grégoire, F.R. Tay, L. Breschi, D.H. Pashley, Water distribution in dentin matrices: Bound vs. unbound water, Dent. Mater. 31 (2015) 205-216.
[2] N. Sagaki, S. Shiwa, S. Yagihara, K. Hikichi, X-ray diffraction studies on the structure of hydrated collagen, Biopolymers 22 (1983) 2539-2547.

[3] L. Tjäderhane, F.D. Nascimento, L. Breschi, A. Mazzoni, I.L. Tersariol, S. Geraldeli, A. Tezvergil-Mutluay, M.R. Carrilho, R.M. Carvalho, F.R. Tay, D.H. Pashley, Optimizing dentin bond durability: Control of collagen degradation by matrix metalloproteinases and cysteine cathepsins, Dent. Mater. 29 (2013) $116-135$.

[4] L. Tjäderhane, F.D. Nascimento, L. Breschi, A. Mazzoni, I.L. Tersariol, S. Geraldeli, A. Tezvergil-Mutluay, M. Carrilho, R.M. Carvalho, F.R. Tay, D.H. Pashley, Strategies to prevent hydrolytic degradation of the hybrid layer: A review, Dent. Mater. 29 (2013) 999-1011.

[5] M.G. Brackett, N. Li, W.W. Brackett, RJ. Sword, Y.P. Qi, L.N. Niu, C.R. Pucci, A Dib, D.H. Pashley, F.R. Tay, The critical barrier to progress in dentine bonding with the etch-and-rinse technique, J. Dent. 39 (2011) 238-248.

[6] L.E. Bertassoni, J.P. Orgel, O. Antipova, M.V. Swain, The dentin organic matrix limitations of restorative dentistry hidden on the nanometer scale, Acta Biomater. 8 (2012) 2419-2433.

[7] G. Grégorie, P. Sharrock, M. Delannée, M.B. Delisle, Depletion of water molecules during ethanol wet-bonding with etch and rinse dental adhesives, Mater. Sci. Eng. C. Mater. Biol. Appl. 33 (2013) 21-27.

[8] D. Toroian, J.E. Lim, P.A. Price, The size exclusion characteristics of type I collagen: Implications for the role of noncollagenous bone constituents in mineralization, J. Biol. Chem. 282 (2007) 22437-22447.

[9] M. Takahashi, M. Nakajima, J. Tagami, D.L. Scheffel, R.M. Carvalho, A. Mazzoni, M. Cadenaro, A. Tezvergil-Mutluay, L. Breschi, L. Tjäderhane, S.S. Jang, F.R. Tay, K.A. Agee, D.H. Pashley, The importance of size-exclusion characteristics of type I collagen in bonding to dentin matrices, Acta Biomater. 9 (2013) 95229528.

[10] A.E. Tami, M.B. Schaffler, M.L. Knothe Tate, Probing the tissue to subcellular level structure underlying bone's molecular sieving function, Biorheology 40 (2003) 577-590.

[11] S.R. Elliott, R.A. Robinson, The water content of bone. I. The mass of water, inorganic crystals, organic matrix, and $\mathrm{CO}_{2}$ space components in a unit volume of the dog bone, J. Bone Joint Surg. Am. 39 (1957) 167-188.

[12] D. Magne, P. Weiss, J.M. Bouler, O. Laboux, G. Daculsi, Study of the maturation of the organic (type I collagen) and mineral (nonstoichiometric apatite) constituents of a calcified tissue (dentin) as a function of location: a Fourier transform infrared microspectroscopic investigation, J. Bone Miner. Res. 16 (2001) 750-757.

[13] N. Hiraishi, N. Tochio, T. Kigawa, M. Otsuki, J. Tagami, Molecular level evaluation on HEMA interaction with a collagen molecule, Dent. Mater. 31 (2015) 88-92.

[14] T.K. Vaidyanathan, J. Vaidyanathan, Recent advances in the theory and mechanism of adhesive resin bonding to dentin: a critical review, J. Biomed. Mater. Res. Part B: Appl. Biomater. 88 (2009) 558-578.

[15] F.R. Tay, R.M. Carvalho, D.H. Pashley, Water movement across bonded dentin too much of a good thing, J. Appl. Oral Sci. 12 (2004) 12-25.

[16] C.M. Hansen, The universality of the solubility parameter, Ind. Eng. Chem. Prod. Res. Dev. 8 (1969) 2-11.

[17] D.W. Van Krevelan, Chapter 7 - Cohesive Properties and Solubility, in: Properties of Polymers: Their Correlations with Chemical Structure, Their Numerical Estimation and Prediction from Additive Group Contributions, Elsevier, New York, 1990, pp. 194-219.

[18] D.H. Pashley, F.R. Tay, M. Carvalho, F.A. Rueggeberg, K.A. Agee, M. Carrilho, A. Donnelly, F. Garcia-Godoy, From dry bonding to water-wet bonding to ethanol-wet bonding. A review of the interactions between dentin matrix and solvated resins using a macromodel of the hybrid layer, Am. J. Dent. 20 (2007) 7-20.

[19] S. Ito, M. Hashimoto, B. Wadgaonkar, N. Svizero, R.M. Carvalho, C. Yiu, F.A. Rueggeberg, S. Foulger, T. Saito, Y. Nishitani, M. Yoshiyama, F.R. Tay, D.H. Pashley, Effects of resin hydrophilicity on water sorption and changes in modulus of elasticity, Biomaterials 26 (2005) 6449-6459.

[20] J. Malacarne, R.M. Carvalho, M.F. de Goes, N. Svizero, D.H. Pashley, F.R. Tay, C.K. Yiu, M.R. Carrilho, Water sorption/solubility of dental adhesive resins, Dent. Mater. 22 (2006) 973-980.

[21] F.T. Sadek, C.S. Castellan, R.R. Braga, S. Mai, L. Tjäderhane, D.H. Pashley, F.R. Tay, One-year stability of resin-dentin bonds created with a hydrophobic ethanol-wet bonding technique, Dent. Mater. 26 (2010) 380-386.

[22] F.T. Sadek, R.R. Braga, A. Muench, Y. Liu, D.H. Pashley, F.R. Tay, Ethanol wetbonding challenges current anti-degradation strategy, J. Dent. Res. 89 (2010) 1499-1504. 